http://dx.doi.org/10.18778/2196-8403.2017.01

TORSTEN VOß

\title{
Die Wiedergeburt der verlorenen Heimat aus dem Geist des Romans. Der Schriftsteller Horst Bienek und Schlesien als synästhetischer Grenzraum
}

Der Beitrag setzt sich mit der synästhetischen Gestaltung von Grenzräumen im Werk Horst Bieneks auseinander. Anhand der Berücksichtigung von Romanen, Gedichten und Essays werden Bieneks Praktiken der Erinnerung als ein kreativer Umgang mit Geschichte verstanden, der es ermöglicht, das Verlorene ebenso lebendig zu halten und zu gestalten, wie auch die historischen Ereignisse der ersten Hälfte des 20. Jahrhunderts aufgrund ihrer Vermittlung - über ganz private Schicksale - an den Rezipienten. Bieneks Gleiwitzer Romane und Gedichte werden dadurch zu einem Stück Erinnerungskultur, die sowohl subjektiv als auch repräsentativ sein kann und zusätzlich über Verfahren der Synästhesie die verlorene Heimat wieder zu reanimieren versucht.

The rebirth of lost homeland through the narrative of the novel. The writer Horst Bienek and Silesia as synesthesia-based borderline

The essay deals with synesthetic constructions of spatial borderlines in the works of Horst Bienek. As an analysis of his novels, poems and essays shows, Bienek's writing can be understood as a creative approach to history. The recording of history makes it possible to give presence to a time that is lost, and it also reveals the events of the first half of the $20^{\text {th }}$ century through the reflection of individual experience. Bienek's novels and poems about Gliwice can be described as a part of a culture of memory, which is both subjective memory, and a representative recording of history. His synesthesiabased aesthetic offers the possibility of revivifying Upper Silesia - the lost homeland of Horst Bienek.

Narodziny utraconej ojczyzny z ducha powieści. Horst Bienek i Śląsk jako synestezyjna przestrzeń graniczna

Artykuł tematyzuje syntestezyjne obrazy obszarów granicznych w twórczości Horsta Bienka. Dzięki analizie powieści, liryki i esejów pisarstwo Bienka zostaje ukazane jako kreatywne podejście do historii. Zapisywanie pamięci pozwala zachować to, co minęło 
i dzięki ukazaniu indywidualnych losów ukazać wydarzenia pierwszej połowy XX w. Gliwickie powieści Bienka a także jego wiersze są częścią kultury pamięci, będącej zarówno subiektywnym wspomnieniem jak i reprezentatywnym zapisem historii. Dzięki stylistyce, posługującej się synestezją ożywiona zostaje utracona ojczyzna, Górny Śląsk Horsta Bienka.

\section{Vorab: Schlesien als Erinnerungsraum und Heimat als (fingier- tes) Axiom?}

\section{Ist Kindheit Erinnerung / oder Erinnerung Kindheit? (Horst Bienek)}

Mit Schlesien lässt sich historisch und kulturell sehr viel assoziieren. Im kollektiven Gedächtnis steht es für eine verlorene Heimat, für eine vielseitige Kultur- und Naturlandschaft im territorialen Grenzbereich ${ }^{1}$, für unwiederbringliche Verluste, aber auch für den Beginn des Zweiten Weltkriegs durch den fingierten Überfall auf den Nachrichtensender Gleiwitz und schlussendlich auch für das Menschheitstrauma Auschwitz. Kaum ein Autor hat sich mit dieser Landschaft als Erinnerungs- und damit als Imaginationsraum so intensiv auseinandergesetzt wie der 1990 verstorbene Romancier und Lyriker Horst Bienek. Seine vierbändige Gleiwitzer Chronik von 1975 bis 1982 - neben Walter Kempowskis berühmter Deutsche Chronik und Uwe Johnsons Jahrestagen wohl die umfangreichste erzählerische Form zwecks Erfassung der Geschichte des 20. Jahrhunderts, um ganz verschiedene Topographien als Narrative zu bemühen - ist hier ebenso zu nennen wie die lyrischen und prosaischen Kindheitserinnerungen Gleiwitzer Kindheit (1976) und Reise in die Kindheit (1988) und die süffisante Novelle Königswald von 1984. In all diesen Werken betreibt Bienek eine teilweise melancholische Reanimation eines verloren gegangenen Ortes und setzt sich mit der Geschichtsträchtigkeit Schlesiens, aber auch Ostpreußens und Böhmens auseinander, die durch den subjektiven Blick des Schriftstellers wieder erfahrbar wird, jenseits von Verklärung und Revision, was sich auch im Schlussmonolog der alten Fürstin von Wetternich ausdrückt, als sie und ihre adligen Freundinnen das heimatliche Schloss Königswald für immer verlassen müssen: „Ich merke, das Alter macht es mir leichter, von hier wegzugehen. Sehr bald werde ich ohnehin alles Irdische verlassen müssen.“ (BIENEK 1984:113) Zugleich liegt darin die Voraussetzung für die imaginative Vergegenwärtigung des Raumes.

1 Grundlegendes dazu in: FrançOIS / SEIFARTH / STRUCK (2007). 
Der Beitrag versucht Horst Bieneks Schlesien-Memorabilien als einen kreativen Umgang mit Geschichte und Heimat zu verdeutlichen, der es schafft, das Verlorene ebenso lebendig zu halten und zu gestalten wie die historischen Ereignisse der ersten Hälfte des 20. Jahrhunderts aufgrund ihrer Vermittlung über ganz private Schicksale. Bieneks Gleiwitzer Romane und Gedichte werden dadurch zu einem Stück Erinnerungskultur, die sowohl subjektiv als auch repräsentativ sein kann. Beide Komponenten sollen dabei ausreichend Berücksichtigung erfahren und zur Diskussion gestellt werden.

Über den Anteil der Fiktion im Prozess der Erinnerung gibt es zahlreiche Arbeiten, sei es durch die Hirnforschung, die kulturwissenschaftliche Gedächtnisforschung, aber auch durch die philologische Erzähltheorie. Namen wie Maurice Halbwachs, Aleida Assmann, WolfGang Iser, PhilipPe LeJeune oder GERHART ROTH tauchen in diesem Zusammenhang immer wieder auf. Glaubt man dem Motto, welches Walter Kempowski einigen Romanen seiner sogenannten Deutschen Chronik vorangestellt hat, nämlich das bekannte Alles frei erfunden!, ist die Geschichte der Familie Kempowski keine Autobiographie oder Chronik, sondern eine literarische Fiktion. Auf den zweiten Blick wird jedoch deutlich, dass der Autor auch in Tadellöser \& Wolff von 1971 (vgl. KEMPOWSKI 1996) mit Mitteln des Fingierens und des Narrativen arbeiten muss, um Erinnerungspraxis zu betreiben und die dort reproduzierten Eindrücke mitteilen, ja um sie plastisch machen zu können. Die Aufgabe der Narration ist in diesem Fall die Wiederherstellung von Erfahrungen und Erlebnissen, die mitunter ereignishaft waren und daher vor allem als signifikante und appellative Bilder im Gedächtnis haften geblieben sind. Um diese in einen Diskurs der Mittelbarkeit, also der Medialität transferieren zu können, bedarf es eines tropologischen und rhetorischen Apparats, welche eben diese memorierten Bilder ausdrücken und für den schreibenden Erinnernden, aber auch für seine Leser rezipierbar machen kann. Die Produktion eben dieser Bildartikulationen ist ein eigenständiger kreativer Akt, der es auch nach Ansicht der Autobiographie-Theorie LEJEUNES völlig ausschließt, die Kultivierung von Fiktionen zu vermeiden. ${ }^{2}$ Doch durch die Nähe eben dieser Fiktionen zu den direkt

2 Neben dem Hauptwerk Der autobiographische Pakt (vgl. LEJEUNE 1994) sei auch auf die kürzlich auf Deutsch erschienene Aufsatzsammlung „Liebes Tagebuch“. Zur Theorie und Praxis des Journals (vgl. LEJEUnE 2014) verwiesen. Der Band stellt einen Querschnitt durch die gesamte literarische Autobiographie- und Tagebuchforschung des französischen Literaturwissenschaftlers dar und reicht von Erzeugnissen der Frühen Neuzeit bis hin zur aktuellen Blogkommunikation im Netz. 
erlebten Eindrücken des Memorierenden gewinnen sie an Authentizität, sie werden der konkret erlebten Familiengeschichte vergleichbar.

In anthropologischer Hinsicht versteht sich Heimat als ein Axiom und als eine - von logischer Beweisführung unabhängige - Grundgegebenheit des Daseins. ${ }^{3}$ Der Verlust von Heimat und dessen Bewältigung wird damit zu einem existentiell relevanten Thema bei chronistisch orientierten und dennoch aus verschiedenen Regionen stammenden Autoren wie Horst Bienek und Walter Kempowski, aber auch bei Christiane Brückner, Günther Grass, Siegfried Lenz oder Ilse Gräfin von Bredow. Kempowski beispielsweise betitelt sein Tagebuch von 1991 über die Rückkehr in die Rostocker Heimat mit dem regional und historisch gebundenen Dialektwort Hamit - Warum wohl? Konnte das Verlorene und auch nach der Wende niemals mehr Einholbare nicht anders ausgedrückt werden als mit einer regional gebundenen Bezeichnung? Wahrscheinlich nicht! Denn Hamit in diesem Sinne existiert nicht mehr für den Autor, weder sprachlich noch in realiter. Kempowski selbst schreibt am 01. Januar 1990 in sein Tagebuch:

Setzen wir das Wort ,Hamit' an die Stelle des abgegriffenen Wortes ,Heimat'. ,Hamit‘ wie die Erzgebirgler sagen. Da fühlt man sich schon ganz anders! Und man hat sie ganz für sich, die theure Heimat, weil alle Welt denkt, man spricht von einem fernen Stern. So fern war sie auch, die Heimat, in den vergangenen vierzig Jahren, fern, aber gegenwärtig. Wir möchten sie gerne berühren, die alte Welt, die der Ort unserer frühen Schmerzen ist. Vielleicht geht von einer Berührung ,Heilung' aus? (KEMPOWSKI 2001:8)

Mit der regionalen Variante Hamit fühlt sich der Tagebuchschreiber „ganz anders“. Denn das spezielle Wort bezeichnet seine spezielle - und verlorene - Heimat oder auch Hamit. Das heute quasi fiktiv gewordene Dialektwort ist konstitutiv für das Narrativ Heimat.

Sie wird also zum Imaginationsobjekt und damit zum Gegenstand aber auch zum Zielpunkt literarischer Kreativität. Horst Bienek selbst hat in seinen Notizbüchern sein Romanprojekt quasi poetologisch begleitet und schreibt in Beschreibung einer Provinz (1983): „Eine neue Art von Heimatroman? Der

3 Vgl. hierzu Bausinger/Köstlin (Hrsg.) 1980; BieneK (Hrsg.) 1985; MeCKLENBURG 1987; MoOSMANN 1980; PotT (Hrsg.) 1986; BERGER 2015; HeRMELINGMEIER 2015. Letztere markiert den originellen Ansatz, das Heimatnarrativ über affektiv und phänomenologisch orientierte Erklärungsmuster (nach BACHELARD) zu erfassen, da diese auf die Nachdrücklichkeit von Erinnerungen hinweisen. 
kritische Heimatroman? Warum nicht. Den Begriff und das Genre rehabilitieren." (BIENEK 1983:12) ${ }^{4}$ Mit der Vermischung von Heimatnarration ${ }^{5}$, Autobiound Historiographie und deren mikroskopische Zuspitzung auf einer privaten Ebene $^{6}$ geht Bienek dieses Vorhaben vor allem im Gleiwitz-Zyklus an, welcher von dem bedeutenden Historiker MARTIN BROSZAT auch als eine ,,zeitgeschichtliche Roman-Tetralogie" bezeichnet wird. ${ }^{7}$ Gerade unter einer solchen Perspektive wird es nicht nur notwendig sein, darauf zu verweisen, dass das Autobiographische nicht mit dem Faktischen zu verwechseln ist. Denn laut LEJEUNE „hat Identität nichts mit Ähnlichkeit zu tun. Die Identität ist eine unmittelbar erfaßte Tatsache, die auf der Ebene der Äußerung akzeptiert oder abgelehnt wird; die Ähnlichkeit ist ein aufgrund der Aussage hergestellter Bezug, der sich endlos diskutieren und nuancieren läßt.“ (LEJEUNE 1994:39) Ebenfalls mit LEJEUNE ,wird der Leser aufgefordert, die Romane nicht bloß als Fiktionen zu lesen, die auf eine Wahrheit des ,menschlichen Wesens' verweisen,

In der österreichischen Literatur wird das Konzept des negativen oder auch schwarzen Heimatromans in radikaler Weise in unterschiedlichen Varianten bei vielen Autorinnen und Autoren aufgegriffen. Unter anderem spitzt dies JOSEF WINKLER in seiner Trilogie Das wilde Kärnten (1979-1982) in visionär-apokalyptischen Bildern mit deutlicher Affinität zur katholischen Mystik zu (vgl. WINKLER 1995). Für eine autobiographische Lesart der Romane und Erzählungen WINKLERS (wobei diese nicht mit historischer Verifizierbarkeit übereinstimmen muss, sondern mit der Prägung durch das bäuerlich-katholische Umfeld) mit Blick auf den Heimatdiskurs plädiert auch der Aufsatz von BENOîT PIVERT (vgl. 2008:178-187).

Vgl. dazu AHRENS (2003). Erinnerung wird dabei selbst als eine narrative Technik verstanden.

6 Ein Autor wie Siegfried Lenz geht in seiner frühen Publikation, der Erzählsammlung So zärtlich war Suleyken von 1955, einen anderen und dennoch ähnlichen Weg wie Horst Bienek oder Walter Kempowski. Er verlässt zwar die Chronologie der Romanzyklen und das unmittelbare Notat des Tagebuchs, bleibt jedoch der Vermittlung von Heimat über ihre Bewohner, ja ihre Typen und Existenzen - wie es im Kempowski-Jargon heißen würde - treu. Um einen Eindruck von seiner Heimat Masuren und seiner Stadt Lück (hier als „Suleyken“) zu geben, wählt der Autor den narrativen Modus der Anekdote, um eine regionale und mentalitätstypische Lebens- und Sprechweise aufzuzeigen. Man könnte sogar von anthropomorphen Dialekten sprechen. Er vermittelt also seine Heimat über ihre Bewohner und deren (mitunter absurd anmutenden) Alltag. (vgl. LENZ 1984)

Vgl. BrosZat (1990:102-111). Der Terminus der Zeitgeschichte wird eng gekoppelt an das Erleben von Geschichte durch die Zeitgenossen und an deren Berichte und Erzählungen darüber. 
sondern auch als Phantasmen, die Aufschlüsse über ein Individuum bringen.“ (LEJEUNE 1994:47) In diesem Fall sogar über mehrere Individuen in spezifischen Situationen und nicht nur über den sich in der Retrospektive artikulierenden allwissenden Erzähler. Vielleicht wäre es auch besser, mit LEJEUNE von Identitäten zu sprechen, die dem Rezipienten anschaulich vermittelt werden. Identitäten, die in ihrer Präsentation Authentizität simulieren und damit Nachvollziehbarkeit von historischer Vergangenheit durch die Narrative ihres Erlebens ermöglichen. Das deckt sich auch mit THOMAS B. AHRENS überzeugender Schlussfolgerung einer

Art Familiensaga, worin zahlreiche Kinder und Jugendliche als herausragende Identifikationsfiguren fungieren. [...] Die autobiographische Fundierung der Figuren ist dabei nicht zu übersehen. Bienek wollte zwar mit keiner seiner Figuren vollständig identifiziert werden, aber er bekannte sich deutlich zum Autobiographischen, das ihm zufolge jedes Kunstwerk brauche, ,um wahr, um überzeugend zu sein'. (AHRENS 2003:170)

Das vollzieht sich gerade über den Akt des Fingierens und anhand von einzelnen markanten Episoden, die sich in Bieneks Romanen immer wieder finden und seine gesamte Werk(-auto-)biographie durchziehen.

\section{Der (Heimat-)Schriftsteller Horst Bienek}

Zunächst sollen einige knappe werkrelevante biographische Informationen des sehr zu Unrecht vernachlässigten Autors Horst Bienek nicht vorenthalten werden: Er wurde am 07. Mai 1930 in Gleiwitz, Schlesien (heute: Gliwice, Polen) geboren. Der Vater war Bahnbeamter (wie bei der Familie Ossadnik in der vierbändigen Chronik), die Mutter war Klavierlehrerin (wie bei der Familie Piontek in den Gleiwitz-Romanen). Er besuchte die Bürgerschule in Oberschlesien; nach dem Einmarsch sowjetischer Truppen wurde Bienek als Demontagearbeiter zwangsverpflichtet. Zuvor erlebte er noch den Todesmarsch von Auschwitz-Häftlingen durch seinen Heimatort. 1946 erfolgte die Übersiedlung nach Köthen; später nach Potsdam. Dort beendete der spätere Schriftsteller die Oberschule und legte 1949 das Abitur ab. Ab 1950 war Bienek Redaktionsvolontär bei der Tagespost in Potsdam und publizierte erste Gedichte. 1950 nahm er am ersten Lehrgang für junge Schriftsteller in Bad Saarow (Vorläufer des Leipziger Literaturinstituts) teil, wurde von JOHANNES R. BECHER lobend erwähnt und galt daher im Sinne der DDR-Kulturpolitik als förderungswürdig. Den Höhepunkt dieser Förderung bildete freilich das Jahr 1951, als Bienek Meisterschüler bei Bertolt Brecht am Berliner Ensemble wurde. Noch 
im gleichen Jahr, genauer gesagt im November 1951, wurde das ehemalige Ensemble-Mitglied wegen antisowjetischer Hetze vom MfS verhaftet und den sowjetischen Besatzungsbehörden überstellt.

Im April 1952 erfolgte eine Verurteilung wegen angeblicher Spionage und versuchtem Sturz der DDR-Regierung zu 25 Jahren Zwangsarbeit. Von Mai 1952 bis Oktober 1955 wurde Horst Bienek im Arbeitslager in Workuta gefangen gehalten. Es ist bezeichnend, dass sich BRECHT weder während des Prozesses noch während der Haftzeit in Workuta für seinen ehemaligen Schüler eingesetzt hat. 1955 kam es schließlich zur Amnestie und Entlassung in die Bundesrepublik Deutschland (wohl auch im Zusammenhang mit den Gesprächen zwischen Adenauer und Chrustschow über die Entlassung der letzten deutschen Kriegsgefangenen in der Sowjetunion). Von 1957 bis 1961 war Bienek Redakteur beim Hessischen Rundfunk in Frankfurt am Main; von 1958 bis 1961 Mitherausgeber der Zeitschrift blätter + bilder und Herausgeber der Buchreihe studio 58. 1960 wurde Bienek ein Aufenthalt in Rom bewilligt mittels des angesehenen Villa-Massimo-Stipendiums. Von 1961 bis 1969 war Bienek zunächst Lektor, dann Cheflektor des Deutschen Taschenbuchverlages (dtv) in München, wo auch ein Großteil des eigenen Werks erscheinen konnte; anschließend überwog die Arbeit als freier Schriftsteller und seit 1966 war Bienek auch als Mitglied des PEN-Zentrums der Bundesrepublik Deutschland tätig. Er folgten zahlreiche Vortragsreisen durch Europa, Amerika und Australien. Darüber hinaus war Bienek Mitglied der Bayerischen Akademie der Schönen Künste und der Deutschen Akademie für Sprache und Dichtung in Darmstadt. Sein literarisches Werk umfasst Lyrik, Romane, Erzählungen und Essays, was auch die vorliegenden Ausführungen einigermaßen zu berücksichtigen versuchen. Vor allem beschäftigen sich Bieneks Texte vornehmlich mit der Kriegs- und Nachkriegszeit sowie der menschlichen Selbstbehauptung gegenüber staatlicher Obrigkeit und sei es auch nur im mikroskopischen Umfeld der bürgerlichen Familie, die versucht, sich auch in all ihren Eigenarten und Schwächen selbst treu zu bleiben. Dafür erhielt der Autor zahlreiche Auszeichnungen, vor allem für seine autobiographische Romantetralogie Gleiwitz. Eine oberschlesische Chronik (1975-82). Diese stellt nicht nur eine dichterische Rekonstruktion der Provinz Oberschlesien mit all ihren regionalen Mentalitäten dar, sondern bildet auch eine Kollision der Kindheitsgeschichte des Autors mit der Weltgeschichte, wodurch deren mehr oder weniger spürbare Auswirkungen auf kleinste soziale Einheiten wie die Familie und Freundschaften deutlich gemacht werden. Horst Bienek starb am 07. Dezember 1990 an den Folgen von Aids. Sein letztes Werk, der Gefangenenbericht Workuta, blieb 
unvollendet und erschien erst 2012, herausgegeben vom Chef-Lektor des Hanser Verlags MichAEL KRÜGER, im Göttinger Wallstein Verlag. Trotz dieser recht beeindruckenden Vita, der Literaturpreise und einer umfangreichen Werkproduktion muss an dieser Stelle jedoch konstatiert werden, dass der Autor Horst Bienek lange Jahre keine allzu umfangreiche Aufmerksamkeit innerhalb der germanistischen Literaturwissenschaft in Deutschland erfuhr. Die polnische und auch amerikanische Germanistik war da schon sehr viel weiter, bis hin zum Erstellen von wissenschaftlichen Qualifikationsschriften. Als zu konservativ, unterkühlt, unkritisch und unexperimentell im Umgang mit literarischer Tradition und Geschichte wurde sein Werk durch eine Forschungsrichtung abgelehnt, deren Erkenntnisinteresse sich eher an den sprachavantgardistischen Prosa-Arbeiten von UwE JOHnSON, GÜNTHER GraSS, ARNO SCHMIDT, PETER WEISS oder REINHARD JIRGL orientierte. Darüber hinaus erfolgte auch von Seiten mancher Literaturkritiken der Vorwurf einer mangelnden Auseinandersetzung mit den Verbrechen des Nationalsozialismus bei einem gleichzeitigen Rückzug auf eine privat-familiäre Ebene zwecks Unterlaufung eines konkreten politischen Diskurses oder einer kritischen Auseinandersetzung mit den Jahrhundertverbrechen, deren marginale Ausläufer in den Gleiwitz-Romanen fast nur auf einer peripher-privaten Ebene fragmentarisch erfasst und nicht in ihrer Tragweite erkannt werden, so die beiläufige Beobachtung des von einem SS-Kommando durchgeführten Überfalls durch die jugendlichen Hochzeitsgäste Ulla Ossadnik und Andreas Freitag auf den Nachrichtensender Gleiwitz im Eröffnungsroman Die erste Polka (1975). In seinem Notizbuch macht Bienek gerade daran seine Erzähltechnik und seine eigene Art der Medialisierung von erlebter Zeitgeschichte fest:

Der Überfall auf den Gleiwitzer Sender müßte zentral sein und zugleich auch den Charakter der Beiläufigkeit haben. Denn wie geschichtsträchtig dieses Ereignis war, konnte damals keiner der Beteiligten ermessen. Das Ganze eher aus der Perspektive des Nichtwissens, der Naivität beschreiben. Vielleicht sollten das die Kinder erleben. Ja, ich muß das aus ihrer Perspektive beschreiben. Und auch diese sollte beschränkt sein. Sie sehen den Überfall, während sie sich in einer Tonröhre verstecken. Dieser einengende Ausschnitt macht alles gewichtiger. (BIENEK 1983:21f.)

Geschichte kann zum Narrativ werden, weil sie aus der vom Erzähler gewählten Perspektive der Jugendlichen konkret erfahrbar geworden ist, vorab aller objektivierenden Auswertung, Kontextualisierung und Bilanzierung. Der Blick aus der Tonröhre auf das Geschehen steht damit auch symbolisch für die eingeschränkte und zugleich kondensierte Erfahrbarkeit und damit auch Erzählbarkeit von Geschichte. Bevor sie zur Historie diskursiviert wird, wird 
diese erlebt und damit auch Teil einer Genese zur Adoleszenz der beiden jugendlichen Beobachter des Überfalls auf den Gleiwitzer Sender, was für den Verlauf von Die erste Polka konstitutiv ist.

Interessanterweise sahen gerade aufgrund dieses subjektivistischen Perspektivismus Philologen und auch Kollegen in Bieneks Werk einen gewissen erzählerischen, sozialkritischen und avantgardistischen Mangel, welcher sowohl den Anforderungen durch die moderne und dissonant-diffundierte Gesellschaft als auch den narrativen Vorgaben der klassischen literarischen Moderne, die eben gerade dadurch die neuen Lebenswirklichkeiten und veränderten Wahrnehmungsmodalitäten adäquat zu erfassen versuchte, nicht mehr gerecht werden könne. Vielleicht liegen aber darin der Reiz und die zahlreiche Leser bis heute faszinierende Authentizität der Texte Bieneks über seine schlesische Heimat, fernab jeglichen Revanchismus. Nach Ahrens schrieb er „die Gleiwitzer Romane, um die Kindheit und somit die Heimat zumindest ,literarisch wiederzugewinnen“ und dadurch als Erinnerung zu fixieren." (AHRENS 2003:169)

\subsection{Gleiwitz als Synästhesie in Prosa und Lyrik Bieneks?}

Mit seiner Gleiwitzer Tetralogie zeichnet sich Horst Bienek als relevanter literarischer Chronist und Autobiograph der neuesten deutschen und polnischen Geschichte in Grenzregionen und in historisch gewordenen Kulturlandschaften aus.

Es ist ein Romanzyklus, welcher eben auch eine auf persönliches und individuelles Erleben ausgerichtete Geschichte der ersten Hälfte des 20. Jahrhunderts ist. Die vier Romane haben durch die detailreiche Nachzeichnung der Entwicklung einer oberschlesischen Familie und ihrer zahlreichen Bekanntschaften während der NS-Zeit und des Zweiten Weltkriegs Resonanz erfahren. Außerdem erfolgt auch ein Großteil der dort betriebenen Memorabilien stark fragmentarisch bzw. episodenhaft, trotz der zeitlich chronologischen Organisation des Romangeschehens durch die deutsche Geschichte von 1939 bis 1945. Das heißt, es werden Momente, Augenblicke und besondere Erfahrungen aus dem Leben einzelner Mitglieder der Familie Piontek und ihrer Freunde und Bekannten herausgenommen, die zwar zumeist dem Alltag entstammen, aber gerade dadurch nachvollziehbare Informationen geben über die Erfahrung von Geschichte und Politik auf einer existentiell relevanten Mikroebene, jenseits aller Abstraktion durch objektivierende Begriffsapparaturen. Sie sollen diese olfaktorischen, visuellen und akustischen Erfahrungen von Geschichte vermitteln. Dass es Bienek genau darum geht, dokumentieren auch seine Aufzeichnungen von 1983. Ein synästhetisches Projekt tut sich kund, wenn der Autor schreibt: 
Die Gerüche: Hüttenwerke, Kokereien, Schlackenhalden. Die Geräusche: Fördertürme der Kohlengruben, Dampfkessel, der nahe gelegene Rangierbahnhof mit den Loks, dem Knallen der Puffer und den Signalpfiffen der Rangierer. Die Vegetation üppig. Jasmin, der in G. stark verbreitet war, die Zäune der meisten Gärten umsäumte, schwerer, süßer Geruch, ist sozusagen die östliche Weißdornhecke. Die Königskerze: meine ganze Kindheit ist erfüllt von der Königskerze, sie wucherte und blühte überall, an den Bahnstrecken entlang, am Rande der Chausseen, auf den Pfaden zwischen den Feldern, an der Klodnitz und überhaupt an den kleinen Bächen. [...] Kindheit und Königskerze ist für mich beinahe eins, noch heute, wenn ich die Augen schließe, sehe ich ihre goldenen Glockenblüten. [...] Im Westen übrigens niemals mehr die Königskerze gesehen. Oder habe ich nicht darauf geachtet. (BIENEK 1983:16)

Diese auf synästhetischen (und auch nur ad memoriam ablaufenden) Wahrnehmungen beruhende Retrospektive liefert eine Auseinandersetzung mit der Vergangenheit, die außerhalb der geschichtswissenschaftlichen Epistemologien und Methodologien liegt. Sie bringt das Vergangene der Gerüche, der Blicke und des Hörens ins Gedächtnis. An all diese sinnlichen Wahrnehmungen sind Erlebnisse geknüpft und mit den Erfahrungen wiederum ein Raum bzw. eine Zeit. Beides wurde durch die sinnlichen Momente generiert und nun auch wieder rekonstruiert. Zugleich verhält es sich mit dieser Remedialisierung über die Narration ausgesprochen ambivalent. Die verschwundene Königskerze, die all diese Begebenheiten in sich zu vereinigen scheint, sei im Westen nicht mehr auffindbar und steht insofern ebenso für die verlorene Heimat wie für die verlorene Kindheit. Ihr Verschwundensein ist aber zugleich die Bedingung für ihre Imagination durch das Narrativ. Nur was in realiter nicht (mehr) vorhanden ist, kann zu einem Projekt der Einbildungskraft und damit auch der Erzählkunst werden. Und damit beschäftigt sich der historiographische Narratologe Horst Bienek in seiner Prosa und Lyrik. Das ist aber bei diesem Schriftsteller nicht nur auf die eigenen Wahrnehmungen reduziert.

Auch entfalten sich in Ansätzen verschiedene Blickwinkel und Perspektiven, in denen der allwissende Erzähler immer wieder versucht, das eigene subjektive Erleben der einzelnen Familienmitglieder und Altersstufen zu erfassen, um auf diese Weise zum Beispiel die Relevanz eines Hochzeitsfestes, einer ersten Polka des jugendlichen Protagonisten Josel mit dem verehrten Mädchen Ulla oder auch dessen erste rebellische Versuche gegenüber dem NS-Alltag durch das eher als Abenteuer erlebte Beobachten und Auskundschaften politischer und militärischer Agitation innerhalb einer kindlichen oder jugendlichen 
Biographie und Erlebniswelt zu verdeutlichen. Für den ersten Roman der Tetralogie (und seine eigentliche Titelfigur) ${ }^{8}$ ist daher auch im Zusammenhang mit dem für Bieneks Werk immer wieder relevanten Aspekt der Grenzlandschaft (sowohl geographisch als auch autobiographisch) festzumachen.

Die erste getanzte Polka steht hier auch für ein vielfältiges und unhintergehbares Initiations- und Liminalitätserlebnis, welches sich auf mehreren Ebenen vollzieht: auf einer erotischen, auf einer familiären, auf einer historischen und schließlich auch auf einer gewalttätigen, bis hin zum Tod des Vaters. Sie alle implizieren den Abschied von der Kindheit, offenbaren die Zufälligkeit und Brüchigkeit der eigenen Existenz und des Lebens sui generis und bereiten damit quasi auch schon den großen Verlust und den Abschied von der Heimat vor. Nach der ersten Polka ist für Josel Piontek nichts mehr so wie früher. Er hat die Riten der Erwachsenen (vor allem das Schnapske-Saufen) ebenso kennengelernt wie den erotischen Kitzel und die Gewaltlust, als er einen besoffenen Feldwebel daran hinderte, die von ihm geliebte Ulla Ossadnik zu vergewaltigen. Dennoch sind diese besonderen Erfahrungen an bestimmte Orte, Spatialitäten und Personen gebunden, die sich auf diese Weise in das Bewusstsein der Akteure eingraben, so der Hochzeitssaal im Oberschlesischen Hof oder der Karussell-Platz bei Josel.

Das hat auch die Akzentuierung verschiedener Erzählperspektiven und Erzählstile in den Romanen über den Piontek- und den Ossadnik-Clan vor dem Hintergrund der Provinz Oberschlesien zur Folge, die durch diese räumliche Konkretisation fassbarer werden. Denn die Akteure sind an ihre Orte und Mitmenschen gebunden - und damit auch an Heimat. Über letzteres urteilten die Germanisten KeITH BulLIVANT und KLAUS BRIEGLEB: „Aus ästhetischer Enge experimentellen Schreibens, das seinen eigenen ,avantgardistischen' Anspruch nicht durchhält, ,erlöst‘ das räumliche Erzählen.“ (BULLIVANT/BRIEGLEB: 1992:336) Man kann dies durchaus als ein Zurück zur Orts- und Familiengeschichte verstehen, was im 19. Jahrhundert bereits durch Autorinnen und Autoren wie GotTFRIED KELLER, MARIE VON EBNER-ESCHENBACH, FERDINAND VON SAAR oder BERTOLD AUERBACH mit ihrer regional bezogenen Prosa und dem Genre der Dorfgeschichte praktiziert wurde. Ähnliches gilt für die Vermenschlichung der Flüchtlingsgeschichte anhand der Präsentation von acht aristokratischen Einzelschicksalen im Mikrokosmos des böhmischen Schlosses Königswald in der gleichnamigen Novelle aus dem Jahr 1984.

Eine Gesamtexegese liefert ÅRSTEIN (1988). 
Mein Beitrag versucht sich teilweise in textnaher Lektüre ausgewählten Romanen dieser Familiengeschichte, die der Autor stets auch als Chronik und somit als eine autobiographisch transformierte Zeitgeschichte verstanden wissen wollte, anzunähern und dabei besondere Aufmerksamkeit auf die erzählerischen Verfahren und die Reflexionen über Geschichte und Erinnerung zu richten und darüber hinaus die Frage zu erörtern, inwieweit Bienek an der Erstellung eines eigenen (mehr oder weniger fiktiven) Kosmos gelegen war, um Geschichte und deren Wirken exemplarisch und erzählerisch transformiert begreifbar zu machen. Insgesamt wird auch über die Rolle von Literatur als einer zentralen Vermittlerin von Geschichte neben der eigentlichen Geschichtsschreibung und Historiographie und deren spezifische Möglichkeiten und Grenzen reflektiert. Schließlich behauptet auch Walter Kempowski zu Beginn von Uns geht's ja noch gold: „Alles frei erfunden!“ (KEMPOWSKI 1975:6) Damit bestätigt er die These des amerikanischen Historikers HAYDEN WHITE, dass auch die Geschichtsschreibung nicht auf Stilisierungen und den Apparat der Tropen verzichten könne, um Geschichte zu vermitteln, einen Appellcharakter gegenüber historischer Bewusstseinsbildung zu leisten und letztendlich auch, um Geschichte zu erzeugen. Dazu müsste man WHITES Argument von den Fiktionalisierungsstrategien innerhalb der Geschichtsforschung stärker berücksichtigen, also dass auch diese - wenn sie erfolgreich sein will - mit den Mitteln des literarischen Stils, des Fingierens und damit der Erzählung zu arbeiten hat. Umgekehrt kann der Roman dann auch einen Beitrag zur Geschichtsschreibung leisten, indem er die von historischen Umbrüchen eigentlich Betroffenen in den Mittelpunkt rückt. Mitunter ist das dem auktorialen/heterodiegetischen Erzähler eher bewusst als seinen Figuren, wenn es über Valeska Piontek am Vorabend des Krieges heißt:

Valeska hätte noch lange so dasitzen und die Nacht über sich aufziehen und untergehen lassen können, sie hätte nicht bemerkt, daß sie umstellt war von Seufzern und Gegenständen, die sie (einmal) irgendwo gekauft und dann in ein Regal gestellt und vergessen hatte, von Spiegeln und Sehnsüchten, von denen sie, wenn sie auf einmal als Bilder vor ihr aufstiegen, erschrecken müßte, von den Steinen ihrer Erinnerung, die sie mühsam aufeinanderschichtete und die immer wieder zusammenfielen; wenn sie sich umsähe, müßte sie bemerken, daß die Dinge schon Risse und Bruchstellen hatten, nur feine Äderchen zeigten das an, die man mit bloßem Auge vielleicht nicht gleich erkennen konnte, die aber nicht mehr wegzubringen waren. (BIENEK 2000:298f.)

Durch die akustische, optische und haptische Aufladung der Vergangenheit und der damit verbundenen Versinnlichung der Erinnerung wird deutlich, dass 
die Gegenstände und das Mobiliar in Valeskas Umgebung eine Art Anthropomorphisierung erfahren, da sie mit den Erfahrungsmomenten ihrer Besitzer verbunden sind. Das Interieur spricht also, wird zur historischen und existentiellen Überlieferungsquelle von Schicksalen.

Und von solchen Schicksalen zu berichten, heißt doch nichts anderes als: Geschichten zu erzählen, die Teil der Gesamt-Geschichte sind und von dieser umgeben bzw. berührt werden, so auch die Geschichten der Pionteks, der Ossadniks, der adligen Damen von Schloss Königswald in Böhmen und schlussendlich auch des einsamen Häftlings in den Zellen und Arbeitsstätten des Gulags von Workuta. Folglich heißt dann auch Horst Bieneks erster Roman von 1968 Die Zelle, was ihn wiederum mit Walter Kempowskis Romandebüt Im Block (1969) verbindet, welcher seiner Haftzeit in Bautzen gewidmet ist. Die Zelle (und er selbst) wird zur kleinsten lebenden und damit einengenden Einheit. Er wird quasi selbst zur Zelle. Die Raumvorstellung wird zur eigenen Lebensvorstellung. Das kann für die Zelle in Workuta ebenso gelten wie für den oberschlesischen Raum, der in seiner sinn- und identitätsstiftenden Kraft vor allem sinnlich (hier: olfaktorisch) wahrgenommen und durch den auktorialen Erzähler memoriert wird, so von Josel Pionteks Tante Lucie nach der Hochzeitsfeier und nach der ersten Polka:

Was für eine Nacht! Und sie sog tief die Luft ein: Riecht ihr denn nicht diesen betäubenden Duft des Lavendels! Was für eine Nacht! Sie ging an die wilden Lavendelsträucher heran, und man hörte nur noch ihr Stöhnen, und dann kam sie zurück und balancierte ein paar Blütenköpfe auf ihren Händen, die sie zerrieb, um dann ihrem blinden Sohn die duftenden Hände unter die Nase zu halten. Was für ein Duft, nicht wahr, was für ein Duft ... so duftet unser Land, wirklich, so duftet nur unser Land, sagte sie. (BIENEK 2000:286)

Bereits die im Spätsommer 1939 vollzogene olfaktorische Vergegenwärtigung einer Heimatlandschaft stellt die Antizipation späterer Erinnerungsprozesse in der Gleiwitzer Tetralogie und auch in anderen Werken Bieneks dar. Dass sie sogar Lucies blindem Sohn die Landschaft vermitteln kann, drückt die Bedeutungssuggestivität sinnlicher Wahrnehmung in Bieneks Heimatdiskurs als zentralen Faktor von Mittelbarkeit aus.

Darüber hinausgehend muss aber auch auf einer epistemologischen Ebene gefragt werden, ob die mikroskopisch-privatistische Transformation von Geschichte in Horst Bieneks Erzählweisen und damit quasi deren Synästhetisierung und Vermenschlichung nicht auch einen Beitrag leisten könnte zur Komplexitätsreduktion von historischen Prozessen, was eventuell die Statistiken, 
Bilanzen und auch hoch komplexen Theoriegebäude der Sozial- und Strukturgeschichte in ihrer erkenntnistheoretischen Ausrichtung auf diese Weise nicht einlösen können / wollen. Den Romanen und Erzählungen käme damit eine ähnliche Aufgabe zu wie dem realistisch-naturalistischen Roman der Jahrhundertwende, welcher ebenfalls die Komplexität von Politik und Geschichte (und deren Auswirkungen) auf einzelne Akteure rekurriert. Während dieser jedoch - trotz der Integration des sogenannten einzelnen Mannes in die Narration - an der objektiven Analyse gesellschaftlicher Verhältnisse interessiert war und sich dabei nicht selten einer naturwissenschaftlichen oder zumindest sozialwissenschaftlichen (Milieu) Legitimation verpflichtet fühlte, schaltet Bienek diese Objektivierungstendenzen scheinbar aus und zentralisiert das Weltgeschehen auf den unmittelbaren und subjektiv erfassten Erfahrungshorizont einer einzelnen Familie. Dadurch wird Geschichte zwar ausgesprochen reduktionistisch und unkomplex rezipiert, fernab einer analytischen Exegese und Bewertung, aber zugleich wird sie durch die stilistisch, narrativ und figurativ bedingte Simulation von Echtheit verstehbar, weil sie auch im Rezeptionsprozess erfahrbar gemacht wird. Inwieweit derlei Übertragungsprozesse in Bieneks Erzählwerken Gleiwitz (1975-1982) ${ }^{9}$, Königswald. Eine Novelle (BIENEK 1984) und in Teilen seiner Lyrik (BIENEK 1976) konsequent vollzogen werden, welche Rolle dabei die Beschreibung der Figuren, das anekdotische Erzählen, das soziale Umfeld der Familie und deren sprachliche Verhaltensweisen spielen, wenn es darum geht, vergangene Erfahrungsräume und Erfahrungsweisen per Erzählung auch in ihrer Sinnlichkeit zu vermitteln, wird ebenfalls untersucht.

Dass Literarisierungen die Leserschaft der Gegenwart an Vergangenheit näher heranbringen können, als die geschichtswissenschaftliche Terminologie und die entfremdete Objektivität einer nur in großen Zusammenhängen sich bewegen wollenden Sozial- und Wirtschaftsgeschichte, ist interessanterweise gerade von fachfremder Seite brillant erkannt und auf den Punkt gebracht worden. Der Naturwissenschaftler, Genetiker und Essayist ERWIN CHARGAFF hat in seinem Aufsatz Abscheu vor der Weltgeschichte bemerkt: „Geschichte als Literatur ist etwas ganz anderes als Geschichte als Wissenschaft.“ (CHARGAFF 2002:83) Das mag banal klingen und zunächst Wasser auf die Mühlen der Objektvitäts- und Bilanzenfetischisten sein. Doch CHARGAFF führt sein State-

9 Die Tetralogie setzt sich bekanntlich zusammen aus den Romanen Die erste Polka (1975), Septemberlicht (1977), Zeit ohne Glocken (1979) und dem Finale über Flucht und Vertreibung aus der oberschlesischen Heimat in Gestalt von Erde und Feuer (1982). 
ment begründend und exemplarisch aus, wenn er schreibt: „Auf allen historischen Werken lastet bald der dicke Staub, besonders in unsern fachmännischen Zeiten; aber von einem Gibbon, einem Macaulay, einem Burkhardt läßt er sich leicht wegblasen." (CHARGAFF 2002:83 f.) Auch hier wird von CHARGAFF ein (nicht unwesentlicher) Gemeinplatz gegenüber der englischen Historiographie bemüht: Sie erzählt Geschichte und macht sie zur Narration. Derlei ist nicht staubig, sondern spannend oder anrührend oder ansprechend. Das macht nicht nur literarische Texte reizvoll, sondern auch Geschichte nachvollziehbar. Die Begründung dafür wird von CHARGAFF bereits einige Seiten zuvor geliefert ${ }^{10}$ :

Jeder, in seiner Art, ein großer Prosaschriftsteller und daher - - ein ,daher', das gewiß Widerspruch erregen wird - - ein bedeutender Historiker. Sie alle schrieben über ihre Stadt, ihr Land, ihr Volk, und waren darauf bedacht, ein Bild davon zu geben, das ihrer eigenen Seele und ihrer eigenen Epoche entsprach. (CHARGAFF 2002:69)

Im Gegensatz zu HAYDEN WHITE, wird von CHARGAFF nicht nur eine ästhetische Erklärung geliefert, die also die Geschichtsschreibung an einen guten Stil bzw. eine überzeugende Narration knüpft, sondern sowohl eine inhaltliche als auch eine psychologische Legitimation offeriert. Über seine Stadt, sein Land und sein Volk, seine Landschaft schreibt auch ganz konkret Horst Bienek und wird damit zu einem räumlichen Erzähler, zum Erzähler einer historischen Grenzlandschaft, die zum Zeugen und schließlich auch synchron zum Vermittler und zum Produkt von Geschichte wird, so auch in der beinahe schon geschichtsphilosophischen und fatalistischen Reflexion des sterbenden PiontekPatriarchen Leo Maria:

Es ist ein verfluchtes Stück Erde...Oh, eine verfluchte Erde...Leo Maria stieß das leise, aber heftig hervor. Wird es denn nicht mal aufhören, daß sich die Menschen wegen dieser verfluchten Erde gegenseitig umbringen... Die Armen haben die Saat in die harte trockene oberschlesische Erde gesteckt und sie mit ihrem Schweiß gedüngt, und die Reichen haben die Frucht genommen. Die Armen sind unter die Erde gegangen, haben gegraben in der Erde des Herrn und die Kohle herausgeschaufelt mit Methan in den Lungen, und die Reichen haben sie verkauft. [...] Und jetzt zerpflügen die Armen die Erde des Herrn mit den Kanonen der Reichen. (BIENEK 2000:373f.)

10 Und mit Historikern von der Antike (Sallust) bis zum 20. Jahrhundert (Mommsen) in Verbindung gebracht, die allesamt von CHARGAFF als brillante Stilisten begriffen werden. 
Die Auslassungen über die Gesellschaftspyramide und ihre Konfrontation mit einer göttlichen Ordnung lasse ich einmal dahingestellt ${ }^{11}$ und verweise eher auf Leo Marias Äußerungen zur Erde. Das kann durchaus doppeldeutig rezipiert werden: Mit der Erde kann zum einen der gesamte Erdkreis gemeint sein, was bereits eine Vorausdeutung auf den anstehenden Weltkrieg implizieren würde. Zum anderen kann es aber auch die immer wieder umkämpfte Erde der Grenzregion Oberschlesiens sein, der Ort der massiven Grenzkonflikte, Territorial- und Machtverschiebungen.

Von dieser Landschaft ist der Autor zu Zeiten der Niederschrift schon lange getrennt, was Bienek immer wieder auch zu einem Thema seiner Gedichte werden lässt, so in dem vierteiligen Langgedicht Gleiwitzer Kindheit, welches vor allem die Erinnerungen an Gerüche, Düfte und Farben auf eindringlichste Weise kultiviert (BIENEK 1976:73-83) und diese ganz unwillkürlich während der Lektüreerfahrungen des gealterten lyrischen Ich aufkommen lässt: „Dann aber plötzlich/das Knacken einer Mandel/der Geruch eines bratenden Fischs in Bunzlau-Porzellan/der Schrei eines Eichelhähers im Labander Wald/Verwischte Bilder zittern über/die Netzhaut.“(BIENEK 1976:73f.) Mit der aus Synästhesien sich zusammenfügenden oberschlesischen Landschaft ist die Kindheit des Autors und auch die des jugendlichen Protagonisten Josel Piontek aus Die erste Polka verbunden. Wie so oft bei der auf verlorene Heimat bezogenen Erinnerungsliteratur kann hier von einem doppelten Verlust gesprochen werden, dessen Bestandteile sich sogar gegenseitig ergänzen. Von den Orten seiner Kindheit, also vom Elternhaus, Gleiwitz und Oberschlesien ist der Autor ebenso getrennt wie von der Kindheit selbst. Beides bedingt sich quasi wechselseitig. Der räumliche und der zeitliche Verlust bespiegeln sich gegenseitig. So wie die Kindheit nicht ein zweites Mal erlebt werden kann, ist auch der Ort, an dem sie erlebt wurde, verloren. Die Unwiederholbarkeit einer früheren Lebensepoche impliziert gleichzeitig die Unerreichbarkeit des Ortes. An dem Ort Gleiwitz wurde die Kindheit verbracht und mit dem Ende der Kindheit verschwand auch der Ort Gleiwitz. Der Verlust der Heimat ist gleichzusetzen mit dem Ende der Kindheit, was übrigens auch von Bienek im Materialienband von 1983 am Bild von der Königskerze festgemacht wurde, da der Ort keinen weiteren Anteil mehr hat an der späteren Entwicklung des Autors. Der Ort kennt ihn nur als Kind. Er selbst hat mit dem Ort nur Erfahrungen aus der

11 Auch wenn dieser Todesmonolog einige der regional spezifischen Arbeitsfelder wie Landwirtschaft und Bergbau aufzählt und daher auch mit dem Heimatdiskurs in Verbindung zu bringen wäre. 
Kindheit verbunden. Später driftet beides einerseits auseinander und andererseits greifen zeitliche und räumliche Ebenen ex negativo ineinander. Das macht nicht nur die Tragik, sondern auch den Reiz und die kreative Chance der Erinnerungsprosa aus, deren Verfahren eben beschrieben werden kann mit der Setzung Zwischen Melancholie und (V-)Erarbeitung: Die Wiedergeburt der verlorenen Heimat aus dem Geist des Romans! Was bedeutet das für BIENEKS Erzähltexte und auch für seine Gedichte? Durch welche Ereignisse wird die Kindheit als prägend dargestellt? Wie wird die verlorene Heimat reanimiert bzw. dargestellt oder auch erstellt, um wie eine Heimat wirken zu können? Warum und auf welche Weise ermöglicht der Roman damit eine Aufgabe der Geschichtsschreibung? Und welche Möglichkeiten sind ihm gegeben, die der klassischen Historiographie womöglich weder gegeben noch gestattet sind?

Nach Thorsten Hinz sind die vier Romane des Gleiwitz-Komplexes eine Möglichkeit, ,das Verborgene, Verdrängte, Abwesende, nichtsdestotrotz aber Wirkungsmächtige sichtbar zu machen. " ${ }^{12}$ Charakteristisch ist hierfür der Erinnerungsstrom des sterbenden Familienoberhaupts Leo Maria Piontek am Ende des ersten Bandes der Tetralogie:

Leo Maria ging in Gedanken die alten Wege, den Weg zur Schule in der Hüttenstraße, den Weg zur Concordia-Grube, den Weg nach Przeschlebie zu den ausgebaggerten Seen, oder den Weg den Fluß hinunter, abertausend Male war er diese Wege gegangen, er wußte, wo die Löcher und Luschen waren, die Steine und Aufrisse, wo in der Sonne zuerst der Teer schmolz, er hatte sich das alles erfühlt mit den eigenen Füßen, denn vom Frühjahr bis zum späten Herbst war er sie barfüßig gegangen. [...] Dieser Weg zum Fluß war mit alten Lindenbäumen gesäumt, die (im Sinne der preußischen Verordnung) ganz unnütz waren, die nur ihren Duft verströmten, der so süß, so stark, so sinnenhaft, so verführerisch war, daß Leo Maria manchmal auf die Bäume hinaufkletterte, bin in die Kronen hinein und sich von diesem Geruch einkreisen und betäuben ließ. (BIENEK 2000: 354f.)

12 Der Artikel von Thorsten Hinz findet sich unter: http://kulturportal-westost.eu/biographien/bienek-horst-2. Eine eigene Poetik dieser Funktion von Literatur - auch mit Blick auf die Vermittlung von Vergangenheit und Geschichte - entwirft Bienek in seinem Gedicht Sagen Schweigen Sagen aus dem Zyklus Die Zeit danach aus dem Jahr 1974 (vgl. BIENEK 1976:107). So heißt es in der zweiten Strophe: „Ohne das Sagen gibt es nichts/wenn ich nicht das/was geschehen ist/sage erzähle oder beschreibe/ist das Geschehen überhaupt nicht geschehen.“ Diese fast transzendentale Sicht auf die objektive Welt korrespondiert jedenfalls mit einer postulierten Abhängigkeit der Geschichte oder des Geschehens vom Erzähler. Geschichte besteht also niemals außerhalb der Narration, die damit Vermittler und Erzeuger zugleich ist und stellt damit das lyrische Korrelat zur Tonröhren-Sicht aus Die erste Polka dar. 
Es wird von der Sinnenhaftigkeit als entscheidenden Modus der Wahrnehmung gesprochen. Sie wird in ihrer Unmittelbarkeit über olfaktorische und haptische Wahrnehmung memoriert und qua Memoria in einen Diskurs der Mittelbarkeit transferiert. Das ist entscheidend, wird doch mit dieser während des Sterbens vollzogenen Memoria auch die Poetik des Autors performativ in Szene gesetzt, die in einer Verzahnung von Erzählen, Erinnern und Erschaffen begründet ist. Mit der Erinnerung des Leo Maria endet auch die Beschaffenheit der alten und gekannten Heimat, die durch den memorierenden Sterbenden ein letztes Mal geradezu allegorisch verkörpert wird. Das Vergangene wird nämlich immer wieder durch die Eingriffe des Gegenwärtigen verdrängt, hier vor allem in Gestalt moderner Medien. Das abstrakte (und staatlich-politisch reglementierte) Medium tritt evasiv an die Stelle der Konkretisation sinnlicher Wahrnehmung bzw. derer Reminiszenz im Moment des Sterbens. Denn synchron erfolgt die Radiomeldung des Oberkommandos der Wehrmacht, die den Einmarsch in Polen nach dem von der SS fingierten Überfall auf den Sender Gleiwitz bekanntgibt und auch vorführt, wie Geschichte in die Heimat einer Grenzregion und ihrer Bewohner eingreift und diese grundlegend verändern kann. Und das Begreifen von historischer Relevanz gelingt hier nur durch die zeitgleich erfolgende Fokussierung auf eine private Ebene, auf den Mikrokosmos der Familien Piontek und Ossadnik.

Auch das geschieht im ersten Roman der Tetralogie, in Die erste Polka (1976), nicht nur räumlich, sondern auch zeitlich. Während der Zweite Weltkrieg beginnt, konzentriert sich der gesamte Roman auf einen Tag und auf ein Ereignis, nämlich auf die Hochzeit Irmas, also der Tochter von Valeska Piontek mit dem ins elterliche Haus einquartierten Soldaten Heiko kurz vor Beginn des Zweiten Weltkriegs. Den anderen Hintergrund bildet der Tod des Familienoberhaupts Leo Maria Piontek im unmittelbaren Anschluss an die Festivitäten. Das Hochzeitsfest wird dadurch fast schon mythologisch bis stereotyp überhöht zu einem Tanz auf dem Vulkan, um einmal dieses abgedroschene Bild zu bemühen. Dadurch ist es möglich, Genaueres über die Planungen und den Ablauf einer typisch oberschlesischen Hochzeitsfeier zu erfahren. Durch die genaue Beschreibung der einzelnen Gäste, ihrer Denk- und Verhaltensweisen wird nicht nur Authentizität garantiert, sondern es versammeln sich die unterschiedlichsten Schicksale auf dem Fest - und damit auch ganz verschiedene Erzählungen, ein Verfahren, welches Bienek auch noch in seiner späteren amüsant-lakonischen Erzählung Königswald (1984) beibehalten wird. 
Das gleichnamige Schloss der hochadligen Familie von Wetternich in Böhmen wird zum Kulminationspunkt von verschiedenen Vertriebenenschicksalen, zu einem Kondensat von sich nur noch im Verlust begreifenden Regionen:

Außer den Wetternichs waren nur Flüchtlinge im Schloß und die Woronzoff war der älteste Flüchtling unter ihnen. Sie hatte Rußland 1917 verlassen [...]. Die Gräfin Posadowsky aus dem Ratiborer Land schaute schweigend vor sich hin und knackte mit den Fingern. [...] Sie spielte, um ihre Nerven zu beruhigen und um zu vergessen. Jene Februarnacht zu vergessen, als sie von der anderen Seite der Oder zusehen mußte, wie das Schloß Posadowsky von den Russen erobert wurde und in Flammen aufging. Die ganze Nacht lang brannte der Himmel, und am nächsten Tag stand schwarzer Rauch über den Trümmern. [...] Die Gräfin Huberta von Dohna strickte. Sie seufzte: Ach, Gottchen, und strickte. Sie war aus Ostpreußen geflohen und hatte nicht viel retten können außer ihren Schmuck, einen Lederbeutel mit geschliffenen Bernsteinen und fünf Knäuel Wolle. (BIENEK 1984:19ff.)

Das böhmische Schloss Königswald ist mehr als nur ein Auffangbecken. Es wird zum Archiv einer für immer verschwundenen Vergangenheit und versucht diese vergeblich bis zum Einmarsch der roten Armee durch die permanente Wiederholung auch alltäglicher Verrichtungen zu bewahren, so wie auch der Romanschriftsteller Bienek es selbst mit seiner Gleiwitzer Chronik vollzieht. Damit steht der Archivcharakter des Schlosses als Potpourri von Schicksalen auch für das Archivverfahren des Schriftstellers, welches über die konkrete Gestalt des Schlosses veranschaulicht werden kann. Rekonstruktion bewahrt etwas, jedoch ohne das Original jemals wieder zu erreichen. Das scheint schon die junge Fürstin Ursela von Wetternich angesichts eines letzten großen Festes auf Königswald kurz vor Kriegsende zu begreifen, wenn sie das Ereignis als Bühne erlebt:

Als Ursela zurückkam, blieb sie wie angewurzelt in der Tür stehen. Es war, als begriffe sie erst jetzt das Geschehen: Königswald von der SS besetzt, die Russen näherten sich von der einen, die Amerikaner von der andern Seite [...]. Und hier im Roten Salon wurde ein Fest gefeiert, wie es prächtiger, sorgloser und ausgelassener nicht in Friedenszeiten hätte gefeiert werden können. Das Grammophon spielte eine Polonaise. Woher hatten die Damen nur die eleganten Kleider? Die meisten von ihnen waren als Flüchtlinge mit einem Koffer oder einer Truhe angekommen oder gar nur mit einem Rucksack und einer Handtasche. Jetzt waren sie gepudert und geschminkt, hatten Schmuck angelegt, einige sogar fesche Hüte aufgesetzt - Ursela schien es, als blicke sie auf eine Bühne. (BIENEK 1984:48)

Die Vergangenheit wird bereits in dieser - Anfang Mai 1945 spielenden - Passage aus dem Geist der Gegenwart und seiner Bedingungen rekonstruiert und erscheint daher der Fürstentochter merkwürdig fiktiv. Zugleich markiert die Textstelle das Verfahren des Autors, die Verschränkung von Rekonstruktion 
und Narration unter stark veränderten Produktionsverhältnissen, was für die Auseinandersetzung mit dem Heimat-Komplex konstitutiv ist und die Arbeit des Schriftstellers Bienek immer wieder begleitet hat und daher für kulturwissenschaftliche Heimat-Forschung wieder interessant gemacht hat.

Auch deswegen erfährt in den letzten Jahren das umfangreiche Werk Horst Bieneks, bis hinein in seinen Nachlass, inzwischen wieder eine langsame Annäherung von Seiten der germanistischen Literaturwissenschaft.

Vor allem der Stellenwert Bieneks als historischer Chronist, die Vermittlung von Geschichte über Narration und die Auffassung von Geschichte als große Narration, stehen dabei ebenso im Mittelpunkt des Interesses wie die Bedeutung Bieneks als Erzähler einer bürgerlichen Familie in der ersten Hälfte des 20. Jahrhunderts. Das ist es, was ihn, trotz aller regionaler Differenzen, auch mit dem Chronik-Projekt von Kempowski verbindet. Durch die Verknüpfung fiktionaler und autobiographischer Elemente, vor allem im Hauptwerk der Gleiwitzer Tetralogie, die dieser aber auch stets in Verknüpfung mit seinen umfangreichen Jugend- und Lagererinnerungen, den vereinzelt erschienenen Novellen wie Königswald (1984) ${ }^{13}$ und der Lyrik als Werkeinheit verstanden und rezipiert wissen wollte, schrieb sich der Autor in die Herzen der Leserschaft hinein. Einer Rezeptionsgemeinde, der die Schrecken der Weltkriege und des Dritten Reichs noch im Gedächtnis waren - und mitunter aus den Herzen einer sowohl ideologiekritischen als auch theorieaffinen und stilexperimentellen Literaturwissenschaft hinaus, die in ihm bisweilen einen konservativen und sich an Anekdoten aufhaltenden Rekapitulator der eigenen Familiengeschichte konstatierten, was wiederum eine reflektierte (und schuldbewusste) Auseinandersetzung mit der Rolle und Funktion der bürgerlichen Gesellschaft innerhalb der NS-Diktatur angeblich relativiert haben sollte. Nach HERMELINGMEIER ist aber ,„Heimat‘ als Wahrnehmungskategorie zu verhandeln [...]

13 So finden sich Figuren wie Milka Piontek (die Wasser-Milka) und die von ihr betreute Gräfin Hohenlohe nicht nur in der Gleiwitzer Chronik, sondern gegen Kriegsende auch auf dem böhmischen Schloss Königswald wieder, was eventuell auch des Autors Sehnsucht nach der Konstruktion von großen Zusammenhängen demonstriert, was nicht nur ein ästhetisches, sondern auch ein existentielles und anthropologisches Anliegen zu sein scheint. Die Errichtung von Zyklizität ermöglicht das Bewahren von Ganzheit. Und Letzteres bildet eine Größe, die der Autor mit der verloren gegangenen Kindheit (= Heimat) untrennbar und unrettbar verbindet. Auch das die Romane begleitende Werkstattbuch Beschreibung einer Provinz leistet dazu ebenso einen Beitrag wie die Gedichtzyklen, die wie lyrische Zuspitzungen der umfangreichen Erinnerungsnarrative konzipiert sind. 
auch der Versuch, den Begriff von seinen politischen Zuschreibungen freizulegen, um seiner ideologischen Aufladung zu entgehen." (HERMELINGMEIER 2015:39) Daher wohl auch die so manchen Rezipienten irritierende Konzentration auf die Anekdote, das persönliche Erleben und die eher phänomenologisch angelegte Wahrnehmung von Welt in der Heimatprosa Bieneks, also die über sensualistische Kategorien erfolgenden „unterschiedlichen Zuschreibungen des Begriffs ,Heimat ' [...] durch ihre affektive Dimension.“ (HERMELINGMEIER 2015:40) Das erschwert freilich politische oder ideologiekritische Exegesen der Heimatliteratur.

Auch Walter Kempowski wurde mit derlei negativen Urteilen bis hin zur gezielten Flucht ins Bürgerlich-Beschauliche, ja ins Anekdotenhafte, konfrontiert. Aus diesem Grund ist lange Zeit die Untersuchung von Einzelaspekten in den Büchern von Horst Bienek mitunter auf der Strecke geblieben. Die Bedeutung der eigenen Familie, aber auch der Schule und anderer Institutionen ist ebenso wenig ausführlich behandelt worden wie die der Kirchen, des Katholizismus und der Religion ${ }^{14}$ sowie der Landschaft im allgemeinen, wodurch sich übrigens synchron auch eine Beschäftigung mit der historisch sich verändernden Position eben dieser Kultur- und Sinnstifter während der nationalsozialistischen Diktatur, aber auch schon der Weimarer Republik oder der frühen Bundesrepublik und des Eisernen Vorhangs, hätte ergeben können. Denn die politischen und sozialen Verhältnisse innerhalb dieser verschiedenen Staatsformen haben in den Romanen und Lyriksammlungen des Autors, exemplarisch widergespiegelt am Schicksal von einzelnen Figuren und Familien, eine Verbindung miteinander erfahren, wodurch Horst Bieneks Gesamtwerk sich der Dimension eines Zyklus annähert. Ihn lohnt es, genauer zu erkunden. Eine sich sehr dialektisch ausgestaltende Ambivalenz ist bei dem Verhältnis von narrativer Historiographie, Autobiographie und der Heimat als Erzählstoff bzw. - Modus jedoch immer wieder zu beachten und auch von Horst Bienek in seinen Notizen auf den Punkt gebracht worden. Über seinen Heimatort Gleiwitz schreibt er:

Gleiwitz, ist es noch mein Gleiwitz, die Stadt meiner Kindheit, so wie ich sie jetzt beschreibe? Ich bin ursprünglich von ihr ausgegangen, ganz akribisch, und ich bin nicht wenig stolz darauf, daß man mit diesen Büchern die Straßen und Plätze und Parks dieser Stadt durchstreifen kann wie mit einem Stadtplan. Und doch hat sie sich in meinem Kopf selbständig gemacht, mit anderem Leben, anderem Geist erfüllt, ist jetzt ganz aus der Phantasie gespeist. (BIENEK 1983:149)

14 Ihrer Bedeutung für das Werk Bieneks hat sich FRÜHWALD (2008:265-279) angenommen. 
Das mutet einerseits beinahe tragisch an. Andererseits erklärt es den besonderen Tenor der Schriften Bieneks und die Abhängigkeit der Erinnerung von den Potentialen der Einbildungskraft, ja von dichterischer Phantasie und den Strategien des Fingierens. Die Wiederentdeckung oder Wiedergeburt der Heimat ist zugleich auch immer ihre Erschaffung. Zwischen diesen Grenzpolen bewegt sich Bieneks Narration von einer Grenzlandschaft. Darin liegt sein die Gattungsgrenzen wiederum überschreitendes Projekt begründet:

Jeder Tag köpft eine Stunde der Kindheit/- ich rück näher den Stuhl/an den Schreibtisch heran/rauch eine Malboro/die Bilder erzittern auf meiner Netzhaut/verwischen/ich will sie festhalten/ich schreibe: [...] ewige Kindheit/wo deine Bilder wachsen aus Rauch und Traum und Flamme. (BIENEK 1975:83)

\section{Auswertung/Synopse}

Der Auseinandersetzung mit der oft verlorenen Heimat im fortgeschrittenen Alter - und das ist bei Bienek und auch Kempowski oft der Fall - macht eines deutlich, trotz aller durch die Sprache der Erinnerung simulierten Authentizität. Günter Kunert hat das in seinen gesammelten Aufzeichnungen pointiert:

Jenseits der Altersgrenze von sechzig Jahren zwar keine Bilanz, aber doch Überdenken dessen, was man tat oder unterließ: Künftig reduzierte Möglichkeiten. Eine Straße, die man vielleicht zum letzten Mal fuhr, eine Stadt auf Niewiedersehen, eine unwiederbringliche Landschaft: Das Leben wird zum Erinnern von Erinnerungen, deren Unwiederholbarkeit unabweislich wird. (KUNERT 2004:158) ${ }^{15}$

Aus der Unwiederholbarkeit wird die Unüberschreitbarkeit einer Grenze deutlich, sowohl in spatialer als auch in temporärer Hinsicht. Grenzraum und Grenzzeit bedingen einander. So muss auch der heranwachsende Josel in den finalen Passagen von Die erste Polka durch den auktorialen Erzähler begreifen:

Josel machte selbst die Tür auf und ging hinaus, ohne sich noch einmal umzusehen. Er ging noch einen Schritt, dann war er von der Dunkelheit aufgesogen. Er war älter geworden in dieser Nacht [also in der Nacht seiner ersten Polka und des SS-Überfalls auf den Nachrichtensender Gleiwitz, T.V.], aber noch nicht alt genug. Er wußte plötzlich, er hatte seine Kindheit hinter sich gelassen, und er drehte sich rasch um, als könne er sie noch einmal mit einem Blick zusammenfassen und von ihr Abschied nehmen, aber sie hatte sich in tausend kleine Teile der Trauer, der Melancholie, des Schmerzes und der Finsternis aufgelöst. Er stieg über den Zaun und ging über das abgeerntete Weizenfeld, die Stoppeln knackten unter seinen Schuhen. (BIENEK 1975:341)

Der Eintrag stammt aus dem Jahr 1992. 
Der Zaun steht geradezu paradigmatisch und stereotyp für die liminale Überschreitung einer Grenze, hinter welche das aufgrund seiner Unwiederholbarkeit Unerreichbare liegt und das durch die jugendliche Initiation des Tanzes und das historische Ereignis synchron, also im Sinne KOSELLECKS (2003) in zwei Zeitschichten, die parallel verlaufen sind, seinen Abschluss gefunden hat. Insofern vermittelt der Roman auch das Erfahren von Zeit in seiner Narration. Und Zeitgeschichte als unmittelbare Erfahrung verknüpft die Individual- mit der Universalhistorie.

Diese allgemeine Erkenntnis ist zugleich die eigentliche antizipierende Ausgangslage, vor deren Hintergrund sich der weitere Romanzyklus bewegt: Heimat und Kindheit werden dadurch zu Fiktionen, die dank literarischer Produktivität (wieder) erstellt werden können, was auch zu einer Relativierung von grenzorientierter Wahrnehmung und Denkweise führen könnte, sowohl räumlich als auch zeitlich. Die Grenze würde dann nicht mehr von einem „harten und eindeutigen Schnitt“ (WOKART 1995:284) reguliert werden, sondern könnte als Konstruktionselement von Begriffen, Imaginationen, Erinnerungen und damit von Narrationen begriffen werden. Für Autoren aus dem bzw. über den Grenzbereich heißt das in einer beinahe dialektischen Konstellation: Durch die Unwiederholbarkeit werden sie quasi fiktiv und damit zu Projekten der Imagination. In ihrer Abwesenheit werden sie durch Prozesse des Fingierens anwesend und bürgen somit für die Qualität des Erzählers, welcher Geschichte als (durch Erzählung) erlebtes Geschehen lebendig werden lässt. So konstatiert auch die alte Fürstin Wetternich nach der Aufgabe ihres böhmischen Schlosses und vor der Flucht in den Westen beinahe versöhnlich: „Was ist Besitz? [...] Nichts! Die Wetternichs haben schon vieles verloren in ihrer Geschichte. Sie haben aber auch vieles gewonnen. Das Leben. Nämlich das Leben! Sie warf die Hände in die Luft!“ (BIENEK 1984:113)

\section{Literatur}

Ahrens, Thomas B. (2003): Heimat in Horst Bieneks Gleiwitzer Tetralogie. Erinnerungsdiskurs und Erzählverfahren. Frankfurt a. M. / Bern / New York u. a.

Årstein, MALFrID (1988): Die erste Polka. Eine Interpretation des Romans auf dem Hintergrund der neuen Heimatliteratur. Bergen, unveröffentlichte Staatsexamensarbeit.

Bausinger, Hermann / Köstlin Klaus (eds.) (1980): Heimat und Identität. Probleme regionaler Kultur. Neumünster.

BERGER, KARINA (2015): Heimat, Loss and Identity. Flight and Expulsion in German Literature from the 1950's to the Present. Bern. 
BIENEK, Horst (1975): Die erste Polka. Roman. München / Wien.

BIENEK, HoRst (1976): Gleiwitzer Kindheit. Gedichte aus zwanzig Jahren. München / Wien.

BIENEK, HoRst (1983): Beschreibung einer Provinz. Aufzeichnungen - Materialien - Dokumente. München/Wien.

BIENEK, Horst (1984): Königswald. Eine Novelle. München/Wien.

BIENEK, Horst (ed.) (1985): Heimat: Neue Erkundungen eines alten Themas. München.

BIENEK, HORST (2000): Gleiwitz. Eine oberschlesische Chronik in vier Bänden. München / Wien.

BIENEK, Horst (2013): Workuta. Hrsg. und mit einem Nachwort von MiCHEL KRÜGER. Göttingen.

Broszat, MARTIN (1990): Eine zeitgeschichtliche Roman-Tetralogie. In: URBACH, TiLman (ed.): Horst Bienek. Aufsätze - Materialien - Bibliographie. München, 102-111.

Bullivant, Keith / Klaus BRIEGleb (1992): Die Krise des Erzählens - ,1968 ' und danach. In: KLAUS BRIEGLEB / SIGRID WEIGEL (eds.): Gegenwartsliteratur seit 1968. Hansers Sozialgeschichte der deutschen Literatur vom 16. Jahrhundert bis zur Gegenwart. München / Wien, Band 12:302-339.

CHARGAFF, ERWIN (2002): Abscheu vor der Weltgeschichte. In: ERWIN CHARGAFF: Abscheu vor der Weltgeschichte. Fragmente vom Menschen. Stuttgart, fünfte Auflage: 66-89.

FRANCOIS, ETIENNE / JÖRG SEIFAHRT / BERNHARD STRUCK (eds.) (2007): Die Grenze als Raum. Erfahrung und Konstruktion. Frankfurt a. M. / New York.

FrüHWald, Wolfgang (2008): Passionsfrömmigkeit: Horst Bienek, Peter Huchel, Tankred Dorst. In: FRÜHWALD, WolfGANG (ed.): Das Gedächtnis der Frömmigkeit. Religion, Kirche und Literatur in Deutschland vom Barock bis zur Gegenwart. Frankfurt a. M. / Leipzig, 265-279.

HERMELINGMEIER, ANNA-LENA (2015): Wahrnehmung von Heimat und Exil-am Beispiel von Texten deutschsprachiger Exilautorinnen und-autoren des 20. Jahrhunderts. München.

KEMPOWSKI, WALTER (1996): Tadellöser \& Wolff. Ein bürgerlicher Roman. München, achte Auflage.

KeMPOWSKI, WALTER (2011): Hamit. Tagebuch 1990. München.

Koselleck, Reinhart (2003): Zeitschichten. Studien zur Historik. Mit einem Beitrag von HANS-GEORG GADAMER. Frankfurt a. M.

KunERT, GÜNTER (2004): Die Botschaft des Hotelzimmers an den Gast. Aufzeichnungen. München/Wien.

LAUbe, ReINHART / VerEnA NOlTE (eds.) (2012): Horst Bienek. Ein Schriftsteller in den Extremen des 20. Jahrhunderts. Göttingen. 
LeJeune, PhILIPPE (1994): Der autobiographische Pakt. Aus dem Französischen von Wolfram Bayer und Dieter Hornig. Frankfurt a. M.

LeJeune, PhILIPPE (2014): „Liebes Tagebuch“. Zur Theorie und Praxis des Journals. Hrsg. von Lutz HAGESTEDT. Aus dem Französischen von Jens Hagestedt. München.

LENZ, SIEGFRIED (1984): So zärtlich war Suleyken. Frankfurt a. M.

MeCKlenburg, Norbert (1987): Die grünen Inseln. Zur Kritik des literarischen Heimatkomplexes. München.

MoOSMANN, ERnEST (1980): Heimat. Sehnsucht nach Identität. Berlin.

PIVERT, BENOÎT (2008): Josef Winkler oder das Gefängnis der Vergangenheit. In: Corbin, Anne-MArie / AsPetsberger, Friedbert (eds.): Tradition und Modernen. Historische und ästhetische Analysen der österreichischen Kultur. Innsbruck (Schriftenreihe Literatur des Instituts für Österreichkunde 19):178-187.

Pott, Hans-Georg (ed.) (1986): Literatur und Provinz. Das Konzept ,Heimat “ in der neueren Literatur. Paderborn.

Winkler, Josef (1995): Das wilde Kärnten. Menschenkind - Der Ackermann aus Kärnten-Muttersprache. Drei Romane. Frankfurt a. M.

WOKART, NORBERT (1995): Differenzierungen im Begriff, Grenze ‘. In: FABER, RICHARD / NAUMANN, BARBARA (eds.): Literatur der Grenze - Theorie der Grenze. Würzburg, 275-298. 